\title{
Performance Modeling of Queuing Techniques for Enhance QoS Support for Uniform and Exponential VoIP \& Video based Traffic Distribution
}

\author{
B.K Mishra \\ Principal, Thakur College of \\ Engineering and Technology, Thakur \\ Village, Kandivali (East), \\ Mumbai-400101
}

\author{
S.K Singh \\ Research Scholar-Mukesh Patel \\ School of Technology Management \\ and Engineering,NMIMS University, \\ Mumbai-400056
}

\author{
Kalpana patel \\ PG Student -Thakur College of \\ Science and Commerce, \\ Thakur Village, Kandivali (East) \\ Mumbai -400101
}

\begin{abstract}
In the near future there will be demand for seamless service across different types of network, so it's a significant issue of how to guarantee the quality of service $(\mathrm{QoS})$ and support a variety of services. One important generalization of the Next Generation Network is it's a queue of network. It is expected that traffic in NGN will undergo both quantitative and qualitative changes. Such networks can model problems of contention that arise when a set of resources is shared. With the rapid transformation of the Internet into a commercial infrastructure, demands for service quality have rapidly developed. This paper gives a comparative analysis of three queuing systems FIFO, PQ and WFQ with different traffic distribution. Different traffic distribution includes constant, uniform and exponential traffic distribution. Packet end to end delay, traffic drop and packet delay variation is evaluated through simulation. Results have been evaluated for uniform and exponential traffic distribution. Result shows WFQ has better quality comparing with other techniques in a voice based services and having minimum traffic drop where as PQ techniques is better in Video based services. Simulation is done using OPNET.
\end{abstract}

Keywords-QoS-Quality of service; NGN-Next Generation Network; FIFO- First-in-first-out; PQ- Priority queuing; WFQ- WeightedFair queuing; VoIP-Voice over Internet Protocol.

\section{INTRODUCTION}

It is desirable to impose some traffic-control policy at a network node which depends only on the external traffic loads on the input and output links, but not on the detailed addressing or distribution of packets from inputs to outputs. It should be possible to guarantee the grade of-service of an input-output connection by controlling the aggregate loads on the input and output. There are three type of traffic distribution possible. It is constant, uniform and exponential. Exponential distribution produces heavy traffic as compared to uniform distribution. Hence the amount of traffic dropped is more in exponential distribution [1].

To offer better quality of service in a network a lot of parameter should be considered such as bandwidth, latency, delay, jitter and packet loss etc. These issues have been discussed extensively in past [2] [3]. There are two types of networks.
- Open Queuing network: It is characterized by one or more sources of job arrivals and correspondingly one or more sinks that absorb jobs departing from the network.

- Close Queuing network: In this type of network job neither enters nor depart from the network. The probability of transition between service centers and the distribution of job service time characterized the behavior of jobs within the network. For each center the no. of servers the scheduling discipline and the size of the queue must be specified. We assume that the scheduling is FCFS and that each server has a queue of unlimited capacity.

Output of a queuing system and various algorithms on performance evaluation has been discussed extensively in past through many research papers [4] [5] [6].

\section{VARIOUS QUEUING TECHNIQUES}

In the generic model a node or a service center represent each resource. A service center may have one or more server associated with it. If a job requesting service finds all the server at a service center busy, it will join the queue associated with the center and a later point in time when a server becomes idle a job from the queue will be selected for service according to some scheduling discipline.

There are many elementary queuing models. Attention is paid to methods for the analysis of these models, and also to applications of queuing models on $3 \mathrm{G}$ and $4 \mathrm{G}$ network. Various queuing disciplines can be used to control which packets get transmitted and which packets get dropped. The queuing disciplines are:

- $\quad$ First-in-first-out (FIFO) queuing.

- $\quad$ Priority queuing (PQ)

- Weighted-Fair queuing. (WFQ)

First In First out (FIFO) is the most basic queuing discipline. This expression describes the principle of a queue or first-come first serve behavior: what comes in first is handled first, what comes in next waits until the first is finished etc. 
In FIFO queuing all packets are treated equally by placing them into a single queue, then servicing them in the same order they were placed in the queue. FIFO queuing is also referred to as First Come First Serve (FCFS) queuing. Although a single FIFO queue seems to provide no QoS features at all, it actually does affect drop, delay, and jitter. Because there is only one queue, the router need not classify traffic to place it into different queues and router need not worry about how to decide from which queue it should take the next packet - there is only one choice. Due to this single queue uses FIFO logic, the router need not reorder the packets inside the queue. With a longer queue, however, the average delay increases, because packets may be enqueued behind a larger number of other packets. In most cases when the average delay and average jitter increases [7].

Priority Queuing assigns multiple queues to a network interface with each queue being given a priority level. A queue with higher priority is processed earlier than a queue with lower priority. Priority Queuing has four preconfigured queues, high medium, normal and low priority queue. Queues are serviced in strict order of queue priority, so the high queue always is serviced first, then the next-lower priority and so on. If a lower-priority queue is being serviced and a packet enters a higher queue, that queue is serviced immediately. This mechanism is good for important traffic, but can lead to queue starvation. If packets arrive in the high queue then priority queuing drops everything its doing in order to transmit those packets, and the packets in other queue is again empty. When a packet is sent out an interface, the priority queues on that interface are scanned for packets in descending order for priority. The high priority queues are scanned first, then the medium priority queue and then so on. The packet at the head of the highest queue is chosen for transmission. This procedure is repeated every time when a packet is to be sent. The maximum length of a queue is defined by the length limit. When a queue is longer the limit packets are dropped [8].

The idea of the fair queuing (FQ) discipline is to maintain a separate queue for each flow currently being handled by the router. The router then services these queues in a round robin manner. WFQ allows a weight to be assigned to each flow (queue). This weight effectively controls the percentage of the link's bandwidth each flow will get. WFQ is a generalization of fair queuing (FQ) [9] [10] [11]. Both in WFQ and FQ, each data flow has a separate FIFO queue.

\section{NETWORK DESIGN AND CONFIGURATION}

Various performance modeling on queuing network and mathematical analysis of getting exact solution has been presented via various research papers in past [12] [13]. The following network design has been taken into consideration to evaluate network performance on various queuing network. As shown in Fig 1, the network model consists of two routers having three kinds of traffic sources, FTP traffic, VoIP traffic and video conferencing traffic. The network has been configured for uniform and exponential traffic distribution and result has been explored for voice and video application using FIFO, PQ, and WFQ queuing discipline.
Fig. 2 and 3, shows a heavy traffic load condition and it uses three routers and four routers respectively. For all the network configuration packet end to end delay, traffic drop and packet delay variation has been measured for different queuing discipline. Performance based on queuing network has been discussed in past using various queuing policy but failed to achieve wide acceptance due to various complexity [14] [15] [16].

The below configurations applied in the OPNET IT Guru Academic Edition and simulated to get results.

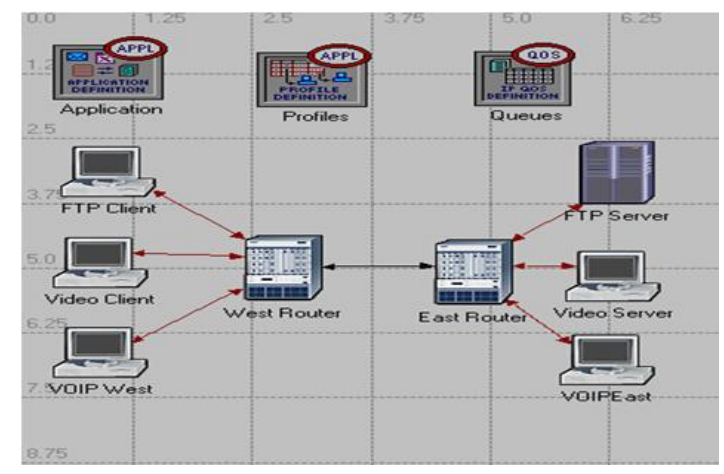

Figure 1. Network Model with 2 Routers

The link connecting the two routers is the bottleneck in the communication. The capacity of this link is $1.54 \mathrm{Mbps}$ whereas all the other links have a capacity of $10 \mathrm{Mbps}$. The following network model is modified by adding one more router to simulate heavy traffic load which is shown in Fig 2. One more router along with three clients is added to the network to increase the traffic load. Further as shown in Fig 3, the number of routers and clients have been almost doubled as compared to the first scenario. Different queuing discipline in the routers can affect the performance of the applications and the utilization of the network resources.

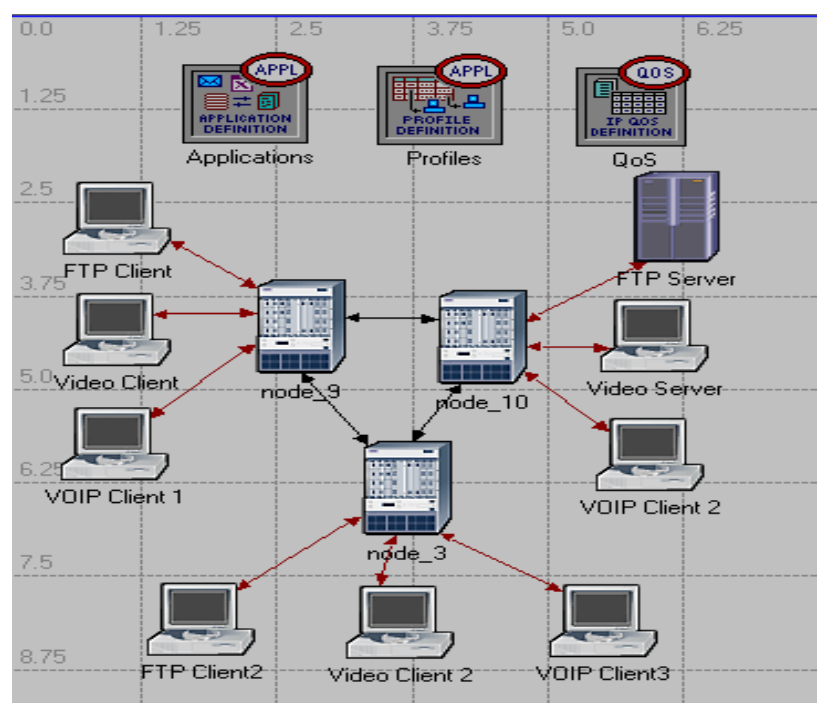

Figure 2. Network Model with 3 Routers 


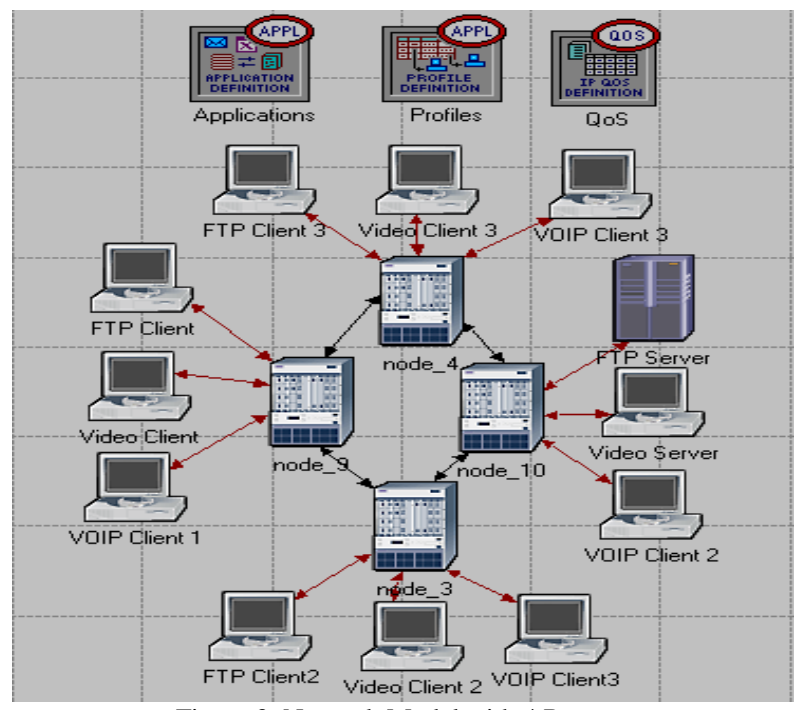

Figure 3. Network Model with 4 Routers

\section{ROUTER CONFIGURATION}

Different queuing discipline in the routers can affect the performance of the applications and the utilization of the network resources. Routers need to be configured for FIFO, PQ and WFQ. The configurations are given Fig 4, 5, 6. Network architecture has been tested for multimedia content delivery and packet end to end delay packet delay variation and traffic dropped has been measured for different queuing discipline.

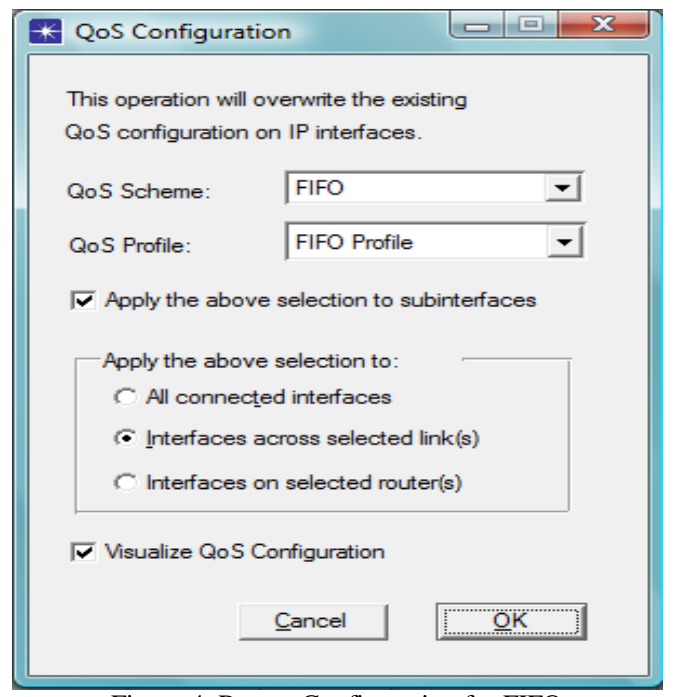

Figure 4. Router Configuration for FIFO

\section{SimULATIONS RESUlTS AND ANALYSIS}

Simulation has been done using OPNET software for every queuing scheme and packet end to end delay packet delay variation and traffic dropped is measured for variable bandwidth. It is tested for voice based and video traffic.

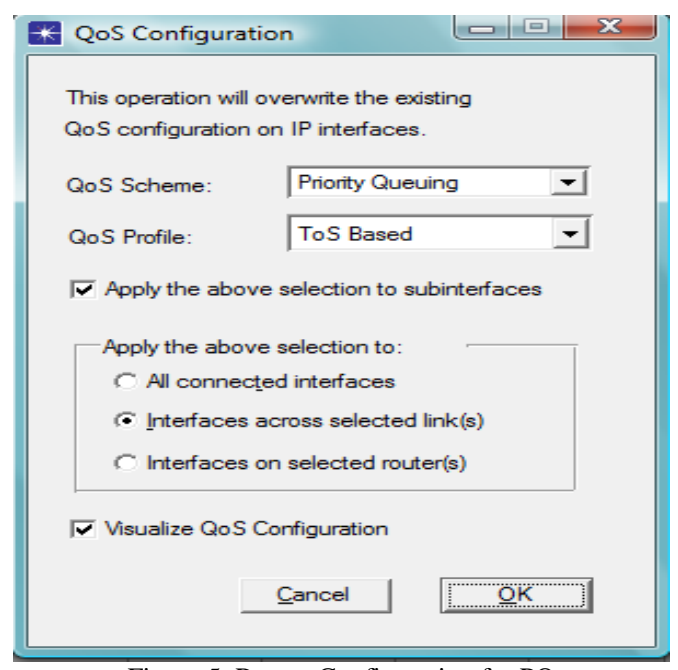

Figure 5. Router Configuration for PQ

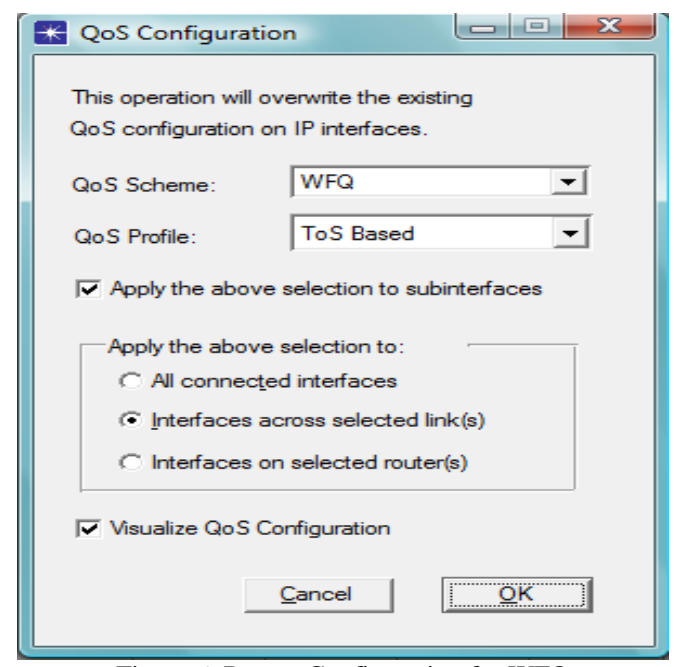

Figure 6. Router Configuration for WFQ

Table 1, 2 and 3 shows statistics for IP traffic dropped, packet end to end delay and delay variation for voice application.

Fig 7, 8, 9 shows individual traffic drop in case of voice transmission for various queuing discipline. Individual traffic drop is nearly zero for WFQ scheme. Individual traffic drop is always higher in case of FIFO scheme Table 1 shows that only packet was drooped when WFQ was implemented whereas 213 packets were dropped for FIFO under two router network model.

Fig 10, 11, 12 shows packet end to end delay in case of voice transmission. Packet end to end delay is nearly zero for both PQ and WFQ scheme. Packet end to end delay is always higher in case of FIFO scheme. From Table 2, packet end to end delay is approximately $744 \mathrm{~ms}$ for FIFO whereas it's just $5 \mathrm{~ms}$ for WFQ.
A. Uniform Distribution Analysis
1) Voice Application 
Table 1 Statistics for IP Traffic Dropped for Uniform Distribution

\begin{tabular}{|c|c|c|c|}
\hline $\begin{array}{c}\text { Number Of } \\
\text { Routers }\end{array}$ & FIFO & PQ & WFQ \\
\hline 2 & 213 & 73 & 1 \\
\hline 3 & 107 & 52 & 1 \\
\hline 4 & 171 & 65 & 1 \\
\hline
\end{tabular}

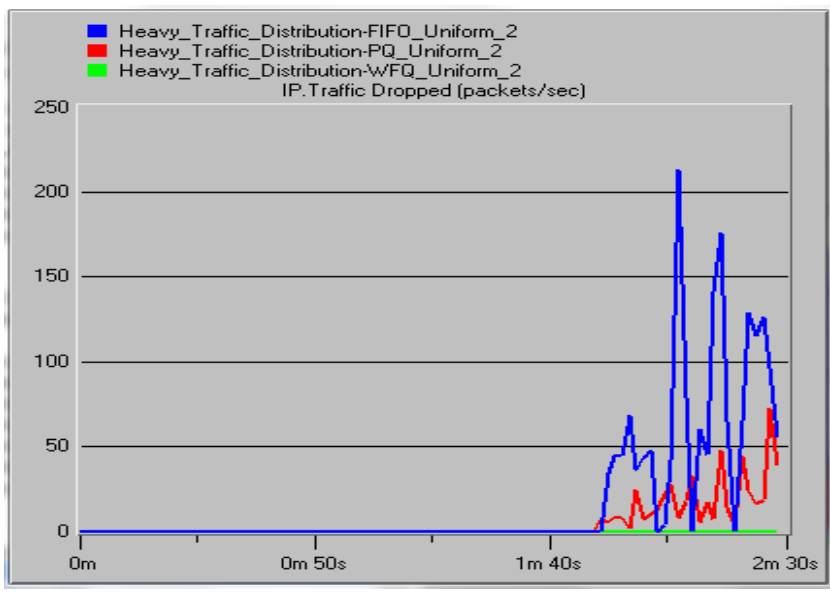

Figure 7. Traffic Dropped for 2 Routers

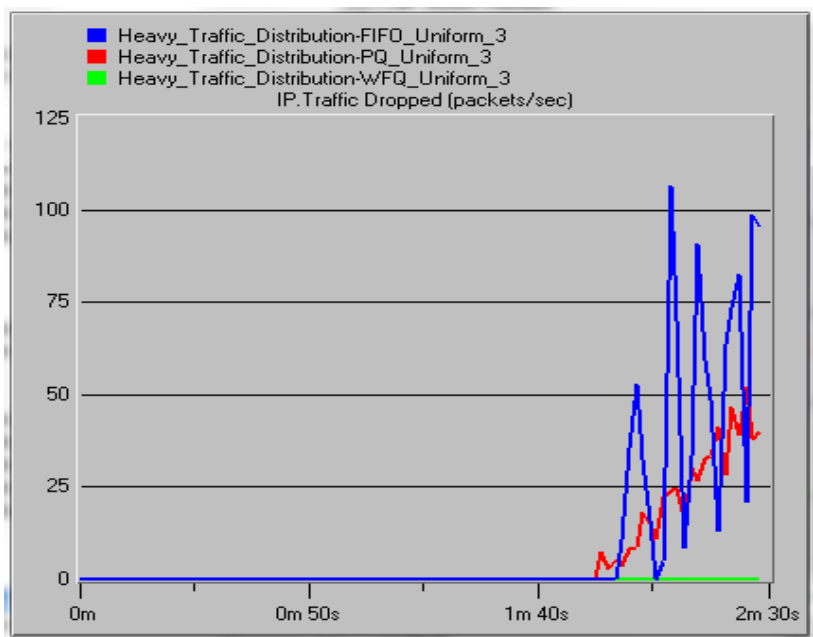

Figure 8. Traffic Dropped for 3 routers

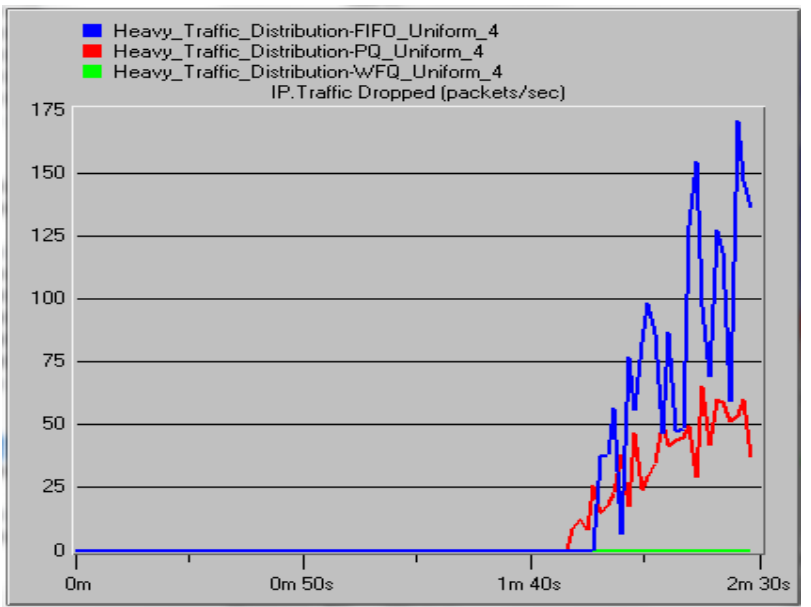

Figure 9. Traffic Dropped for 4 Routers

Table 2. Statistics for Packet End to End Delay for Uniform Distribution

\begin{tabular}{|c|c|c|c|}
\hline Voice & \multicolumn{3}{|c|}{ Packet End to End Delay (Sec) } \\
Applications & FIFO & PQ & WFQ \\
\hline 2 Routers & 0.744296 & 0.00433287 & 0.00599595 \\
\hline 3 Routers & 0.454643 & 0.00249817 & 0.00362311 \\
\hline 4 Routers & 0.229768 & 0.0029926 & 0.00412265 \\
\hline
\end{tabular}

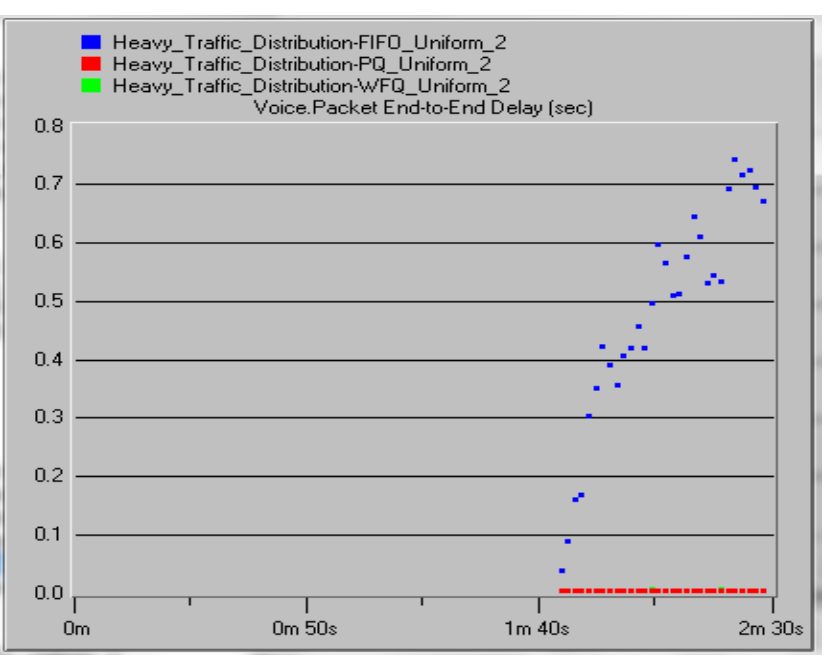

Figure 10. Packet end to end delay 2 Router network configuration

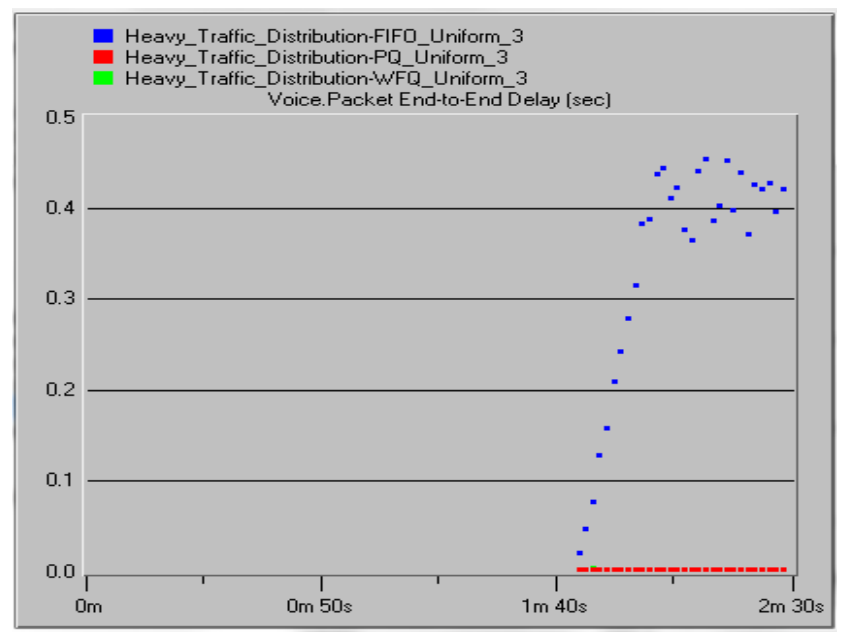

Figure 11. Packet end to end delay for 3 router configuration model.

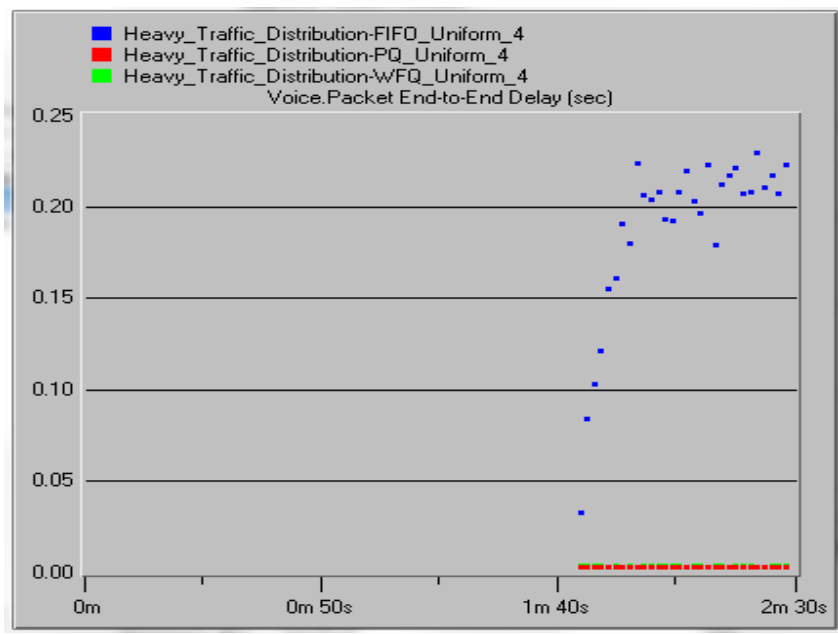

Figure 12. Packet end to end delay for 4 Router configuration model. 
Table 3 Statistics for Packet Delay Variation for Uniform Distribution

\begin{tabular}{|c|c|c|c|}
\hline \multirow{2}{*}{$\begin{array}{c}\text { Voice } \\
\text { Applications }\end{array}$} & \multicolumn{3}{|c|}{ Packet Delay Variation (Sec) } \\
\hline & FIFO & PQ & WFQ \\
\hline 2 Routers & 0.0694031 & 0.0000059404 & 0.0000339073 \\
\hline 3 Routers & 0.0715849 & 0.000003642 & 0.0000208609 \\
\hline 4 Routers & 0.0143389 & 0.0000057815 & 0.0000240066 \\
\hline
\end{tabular}

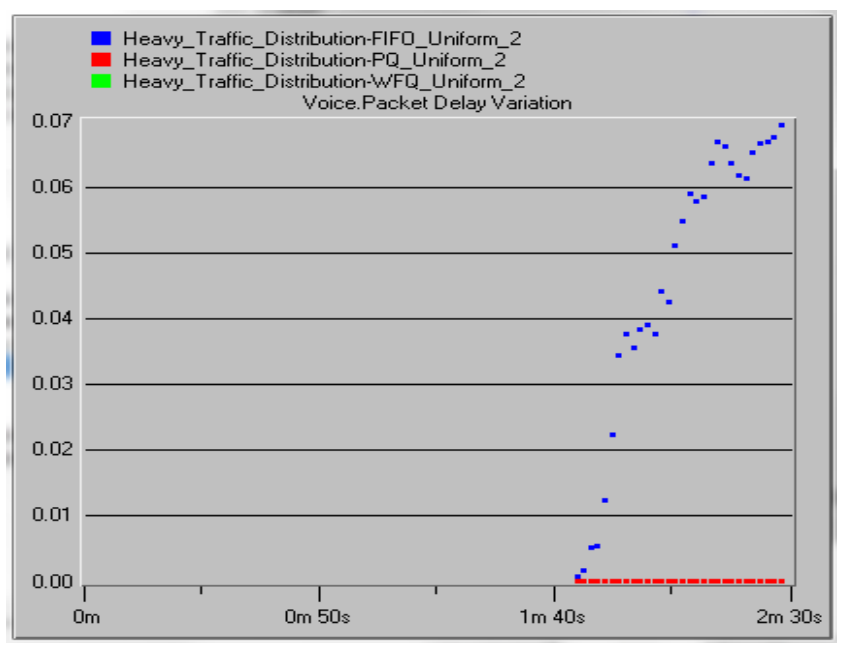

Figure 13. Packet delay variation 2 Router configuration model.

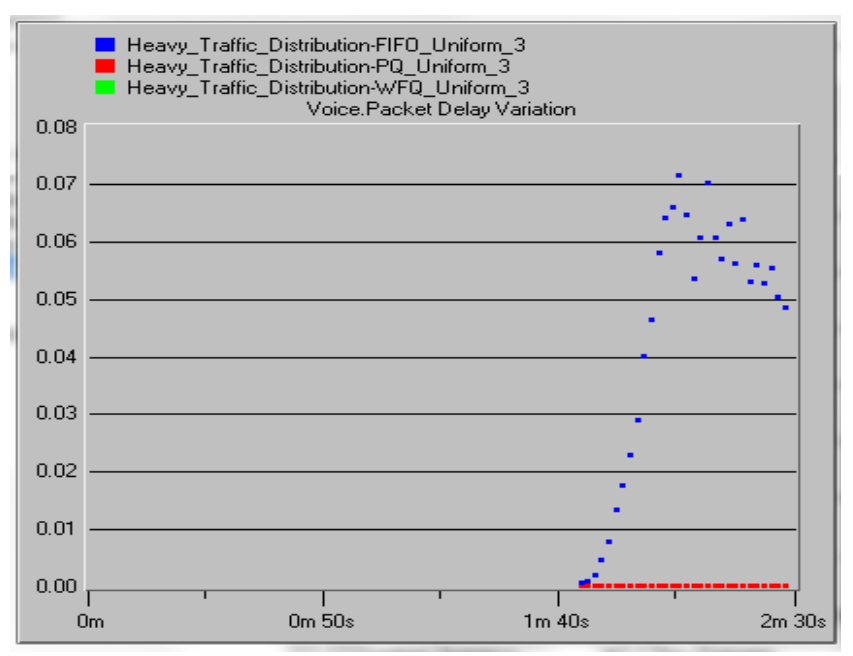

Figure 14. Packet delay variation for 3 Router configuration model.

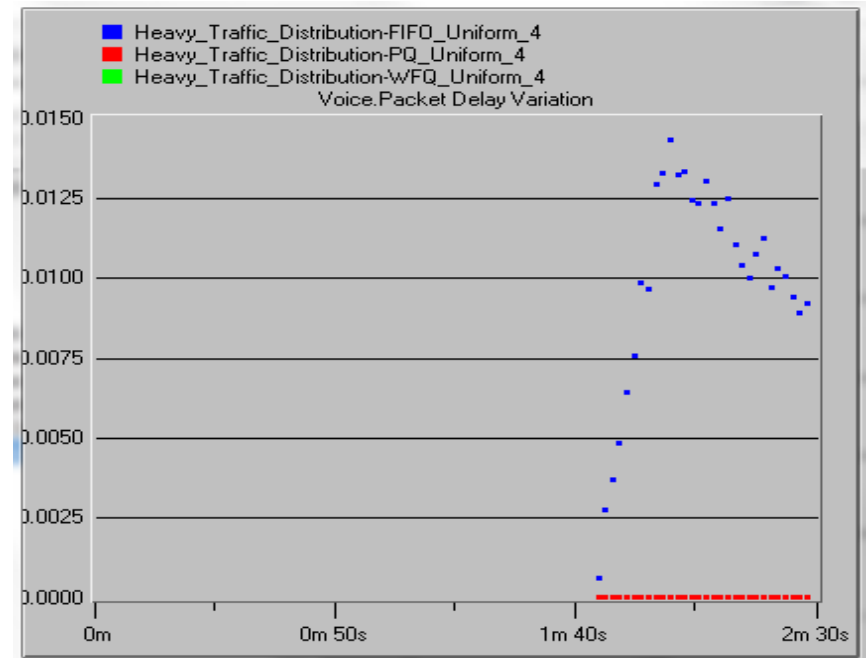

Figure 15. Packet delay variation for 4 Router configuration model.

Fig 13, 14, 15 shows packet delay variation of VoIP transmission for two router, three router and four router configuration model. Packet delay variation is nearly zero for both PQ and WFQ scheme. Packet delay variation is always higher in case of FIFO scheme. It comes out to be $69 \mathrm{~ms}$ whereas $0.03 \mathrm{~ms}$ for WFQ as shown in Table 3.

\section{2) Video Application}

Table 4. Statistics for Packet End to End Delay for Uniform Distribution

\begin{tabular}{|c|c|c|c|}
\hline $\begin{array}{c}\text { Video } \\
\text { Application }\end{array}$ & \multicolumn{3}{|c|}{ Packet End To End Delay (Sec) } \\
& FIFO & PQ & WFQ \\
\hline 2 Routers & 0.84052 & 0.114292 & 0.11387 \\
\hline 3 Routers & 0.764052 & 0.103841 & 0.104795 \\
\hline 4 Routers & 1.34993 & 0.13636 & 0.132879 \\
\hline
\end{tabular}

Table 4, 5 shows statistics for packet end to end delay and delay variation for video application.

Fig 16, 17, 18 shows packet end to end delay for all the three network architecture. Packet end to end delay is nearly zero for both PQ and WFQ scheme. Packet end to end delay is always higher in case of FIFO scheme. From Table 4, packet end to end delay is approximately $840 \mathrm{~ms}$ for FIFO whereas it's $113 \mathrm{~ms}$ for WFQ and $114 \mathrm{~ms}$ for PQ which is quite less as compared to FIFO. 


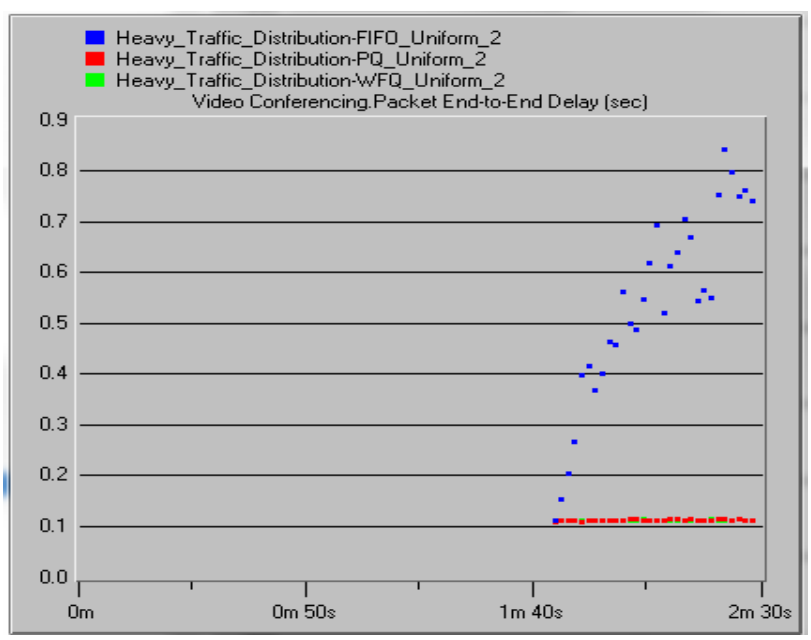

Figure 16. Packet end to end delay 2 Router network configuration

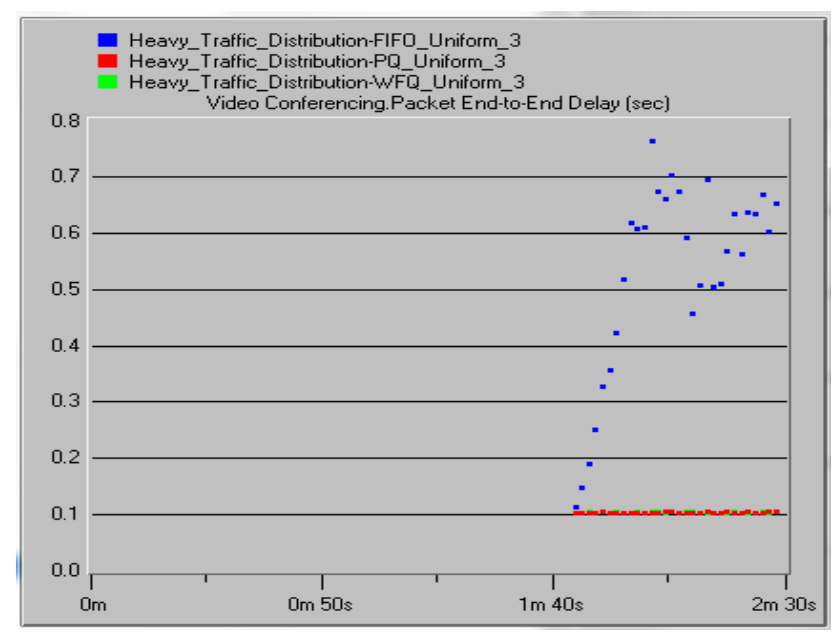

Figure 17. Packet end to end delay 3 Router network configuration

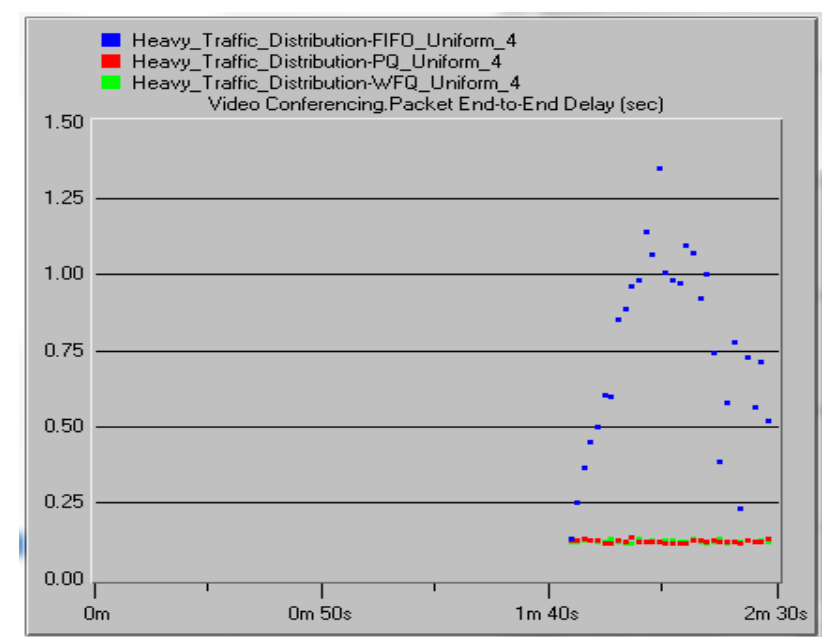

Figure 18. Packet end to end delay 4 Router network configuration
Table 5. Statistics for Packet Delay Variation for Uniform Distribution

\begin{tabular}{|c|c|c|c|}
\hline $\begin{array}{c}\text { Video } \\
\text { Application }\end{array}$ & \multicolumn{3}{|c|}{ Packet Delay Variation (Sec) } \\
& FIFO & PQ & WFQ \\
\hline 2 Routers & 0.083246 & 0.0000092384 & 0.000016311 \\
\hline 3 Routers & 0.209997 & 0.0000556291 & 0.0000540397 \\
\hline 4 Routers & 1.18769 & 0.00227008 & 0.00207855 \\
\hline
\end{tabular}

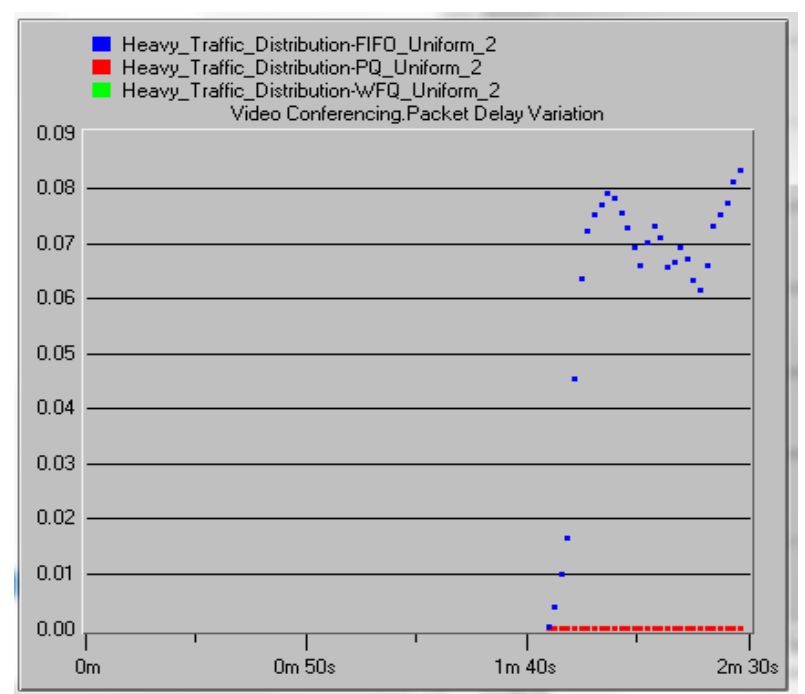

Figure 19. Packet delay variation for 2 Router configuration model.

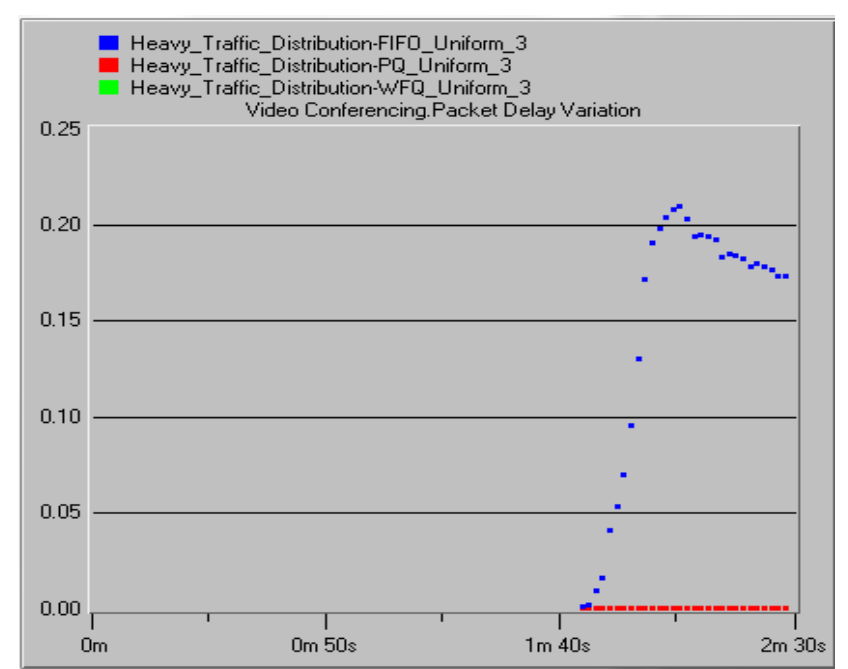

Figure 20. Packet delay variation for 3 Router configuration model.

Fig 19, 20, 21 shows packet delay variation of Video transmission for two router, three router and four router configuration model. Packet delay variation is nearly zero for both PQ and WFQ scheme. Packet delay variation is always higher in case of FIFO scheme. It comes out to be $83 \mathrm{~ms}$ whereas $0.01 \mathrm{~ms}$ for WFQ and $0.009 \mathrm{~ms}$ for PQ as shown in Table 5 . 


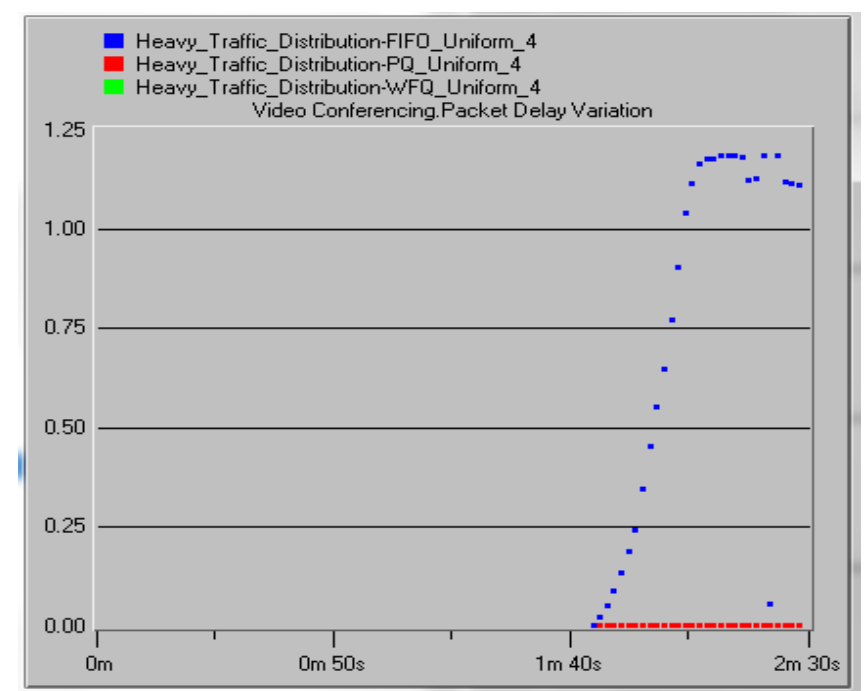

Figure 21. Packet delay variation for 4 Router configuration model.

\section{B. Exponential Traffic Distribution Analysis}

As Exponential distribution produces heavy traffic as compared to uniform distribution so the amount of traffic dropped is more in exponential distribution as shown in Fig 22, 23, 24 for all the three network model. Individual traffic drop is less for WFQ scheme.

Individual traffic drop is always higher in case of FIFO scheme.

Table 6, 7 and 8 shows statistics for IP traffic dropped, packet end to end delay and delay variation for voice application.

Fig 25, 26, 27 shows packet end to end delay in case of voice transmission. Packet end to end time delay is nearly zero for both PQ and WFQ scheme. Packet end to end delay is always higher in case of FIFO scheme.

\section{1) Voice Application}

Table 6. Statistics for IP Traffic Dropped for Exponential Distribution

\begin{tabular}{|c|c|c|c|}
\hline $\begin{array}{c}\text { Number Of } \\
\text { Routers }\end{array}$ & FIFO & PQ & WFQ \\
\hline 2 & 579 & 163 & 70 \\
\hline 3 & 409 & 281 & 120 \\
\hline 4 & 821 & 515 & 519 \\
\hline
\end{tabular}

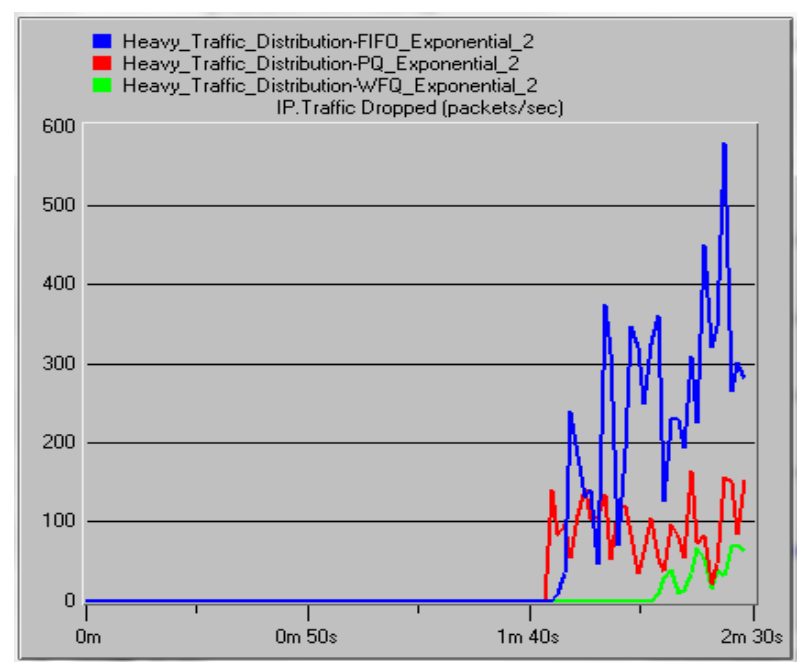

Figure 22. Traffic drop for 2 Router configuration model

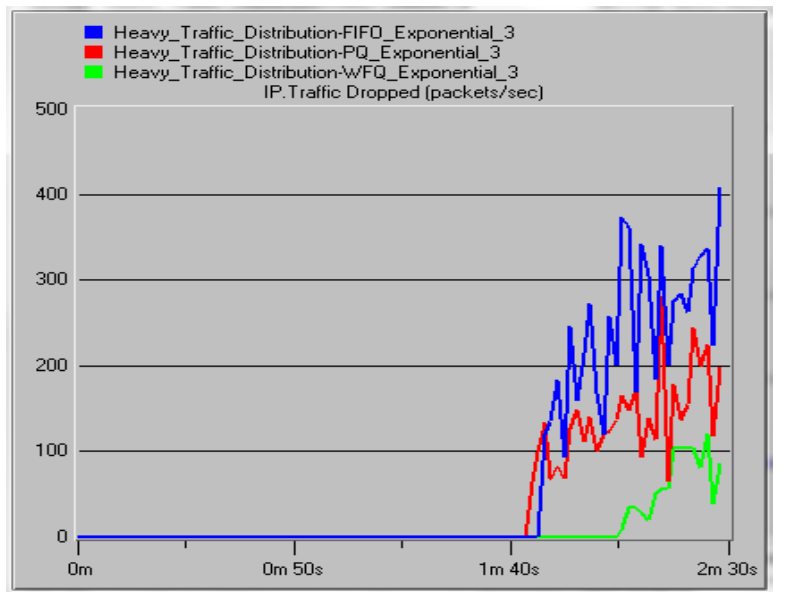

Figure 23. Traffic drop for 3 router configuration network model.

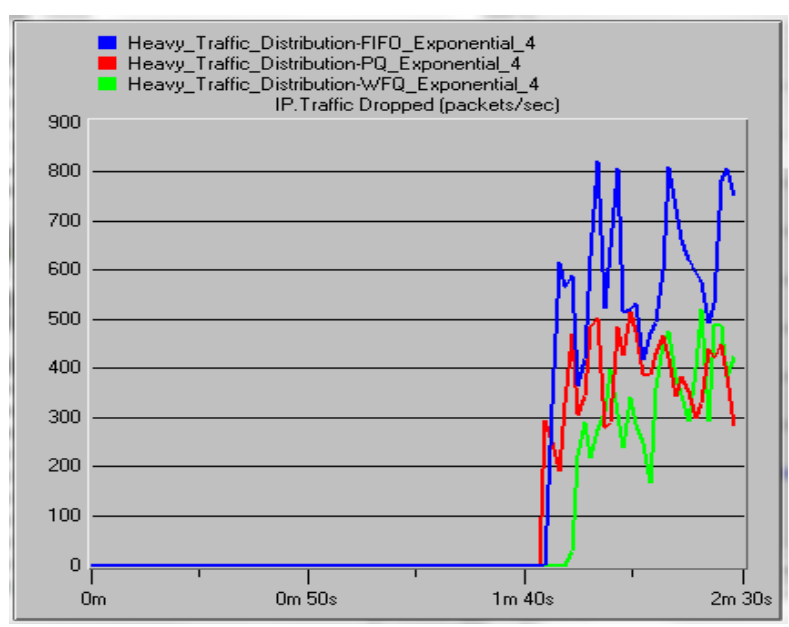

Figure 24. Traffic drop for 4 router configuration network model. 
Table 7. Statistics for Packet End to End Delay for Exponential Distribution

\begin{tabular}{|c|c|c|c|}
\hline Voice & \multicolumn{4}{|c|}{ Packet End To End Delay (Sec) } \\
Applications & FIFO & PQ & WFQ \\
\hline 2 Routers & 0.989209 & 0.00427783 & 0.00509089 \\
\hline 3 Routers & 0.735591 & 0.00302299 & 0.00325629 \\
\hline 4 Routers & 0.539466 & 0.0031793 & 0.00319812 \\
\hline
\end{tabular}

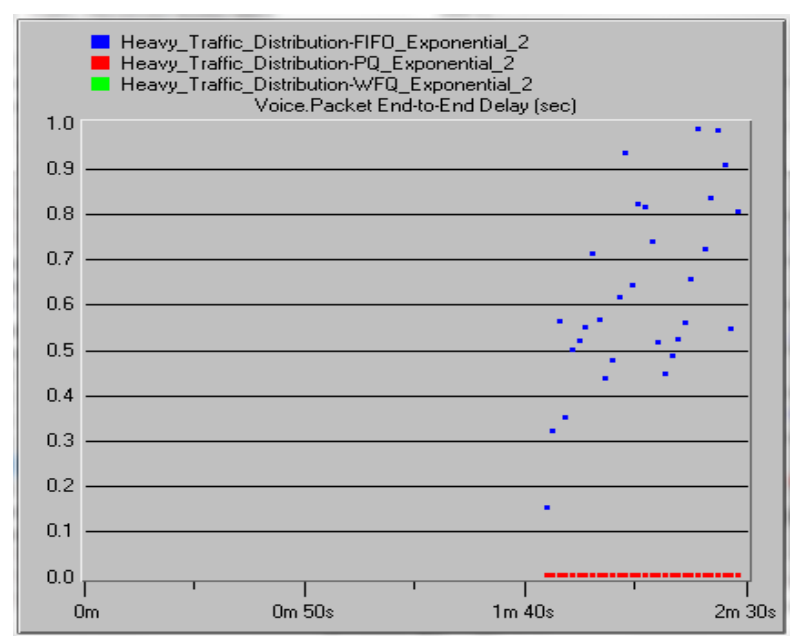

Figure 25. Packet end to end delay for 2 router configuration model.

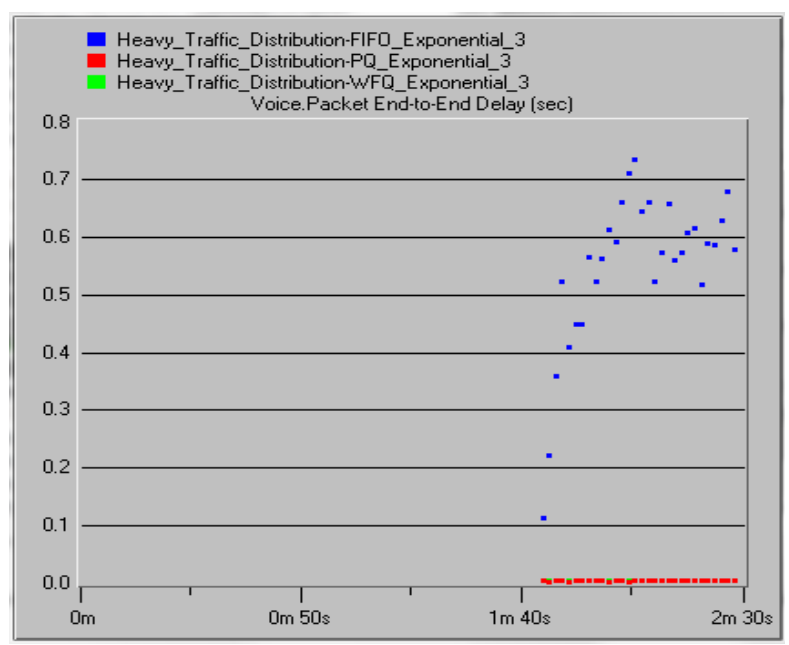

Figure 26. Packet end to end delay for 3 router configuration model.

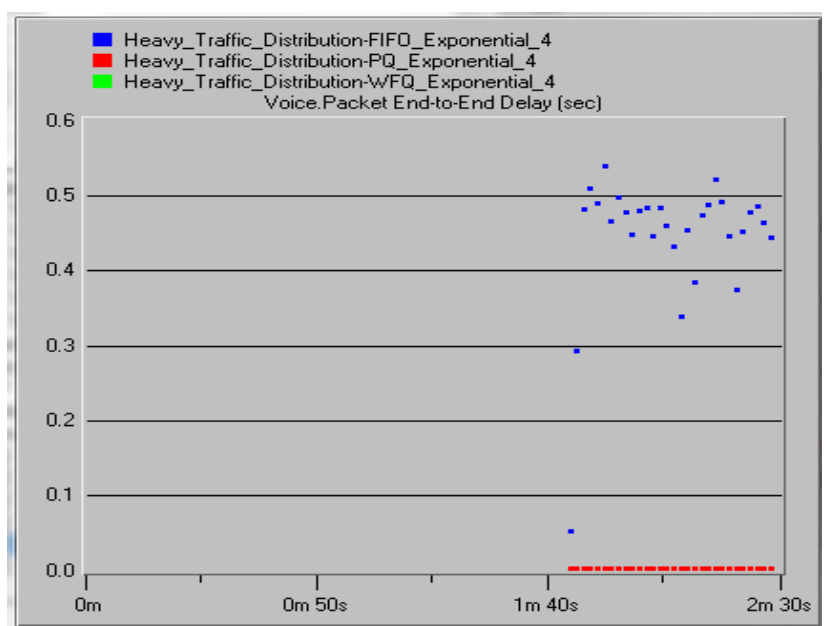

Figure 27. Packet end to end delay for 4 router configuration model.

Table 8. Statistics for Packet Delay Variation for Exponential Distribution

\begin{tabular}{|c|c|c|c|}
\hline $\begin{array}{c}\text { Voice } \\
\text { Applications }\end{array}$ & \multicolumn{3}{|c|}{ Packet Delay Variation (Sec) } \\
FIFO & PQ & WFQ \\
\hline 2 Routers & 0.081209 & 0.0000054636 & 0.0000115066 \\
\hline 3 Routers & 0.112362 & 0.000002987 & 0.0000115894 \\
\hline 4 Routers & 0.0989509 & 0.0000044536 & 0.0000096026 \\
\hline
\end{tabular}

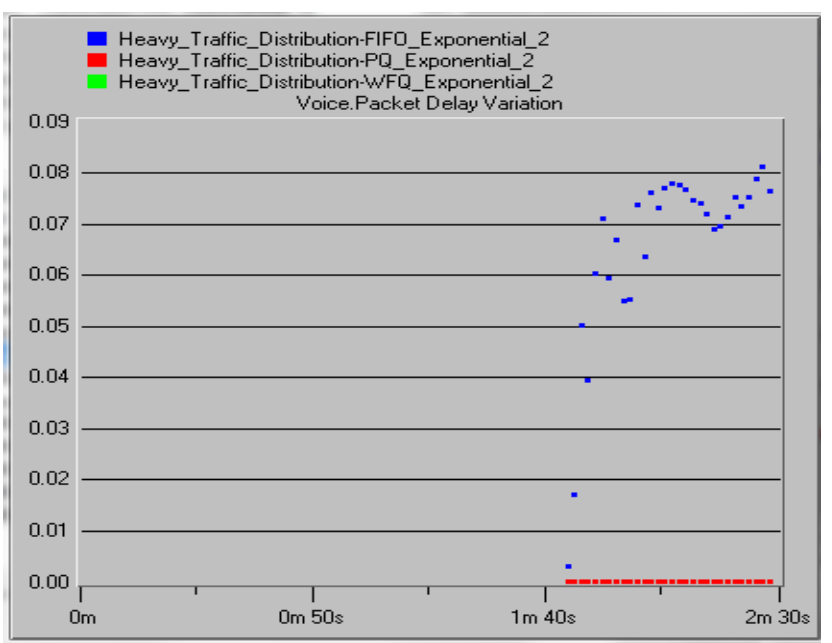

Figure 28. Packet delay variation for 2 router configuration model. 
Packet delay variation is always higher in case of FIFO scheme.

Fig 28, 29, 30 shows packet delay variation of VoIP transmission for two router, three router and four router configuration models. Packet delay variation is nearly zero for both PQ and WFQ scheme.

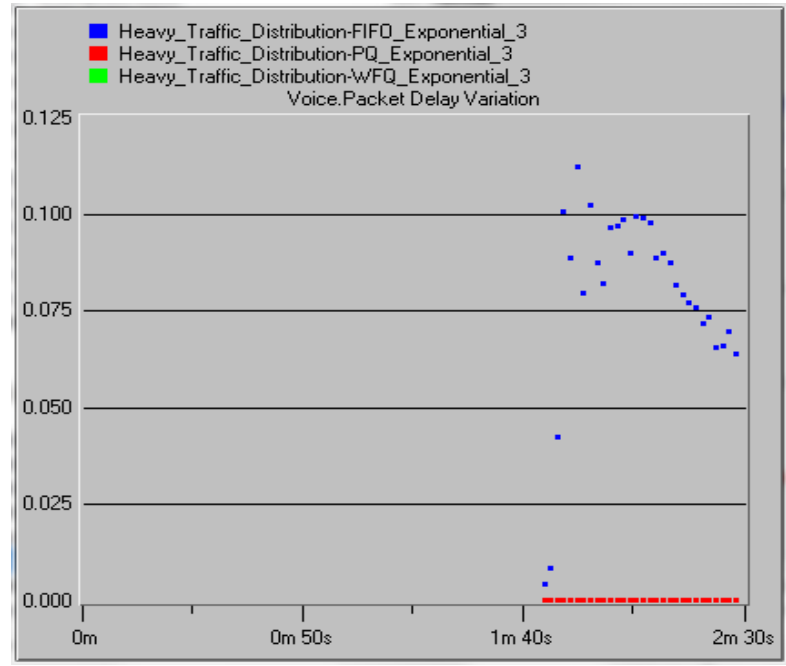

Figure 29. Packet delay variation for 3 router configuration model.

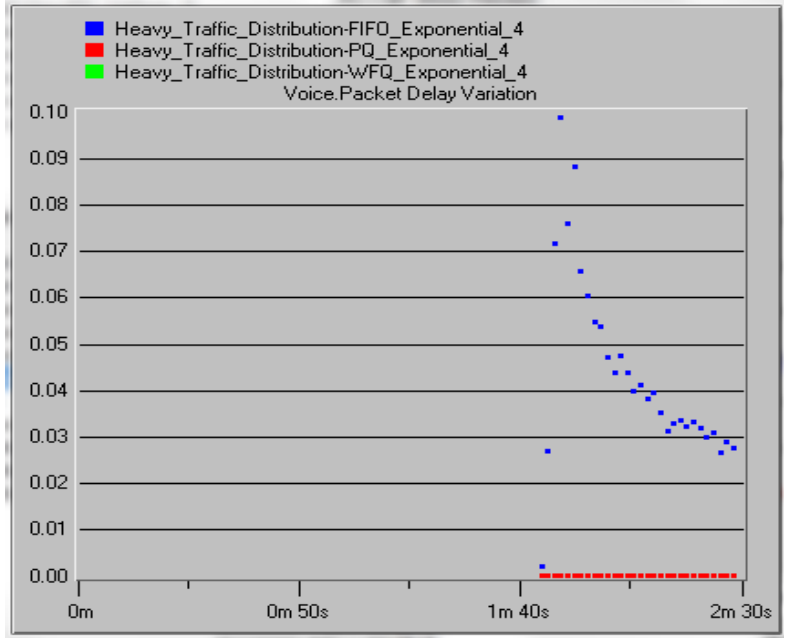

Figure 30. Packet delay variation for 4 router configuration model.

\section{2) Video Application}

Table 9, 10 shows statistics for packet end to end delay and delay variation for video application under Exponential Distribution.

Fig 31, 32 and 33 shows packet end to end delay for all the three network architecture. Unlike voice application, end to end delay comes out be much higher for video application when WFQ was implemented as compared to other two queuing algorithms. It more as video packets are transferred frame by frame and frames are nothing but still images having larger size.
So router takes more time to process these video packets there by increasing overall processing and queuing delay.

With $\mathrm{PQ}$, video packets are transferred with higher priority. Thus end to end delay for PQ is less, it's almost 284 ms as shown in Table 9.

Table 9. Statistics for Packet End to End Delay Exponential Distribution

\begin{tabular}{|c|c|c|c|}
\hline \multirow{2}{*}{$\begin{array}{c}\text { Video } \\
\text { Applications }\end{array}$} & \multicolumn{3}{|c|}{ Packet End To End Delay (Sec) } \\
& FIFO & PQ & WFQ \\
\hline 2 Routers & 1.03799 & 0.284392 & 2.3843 \\
\hline 3 Routers & 1.15523 & 0.224697 & 1.9307 \\
\hline 4 Routers & 1.58502 & 0.265281 & 2.86008 \\
\hline
\end{tabular}

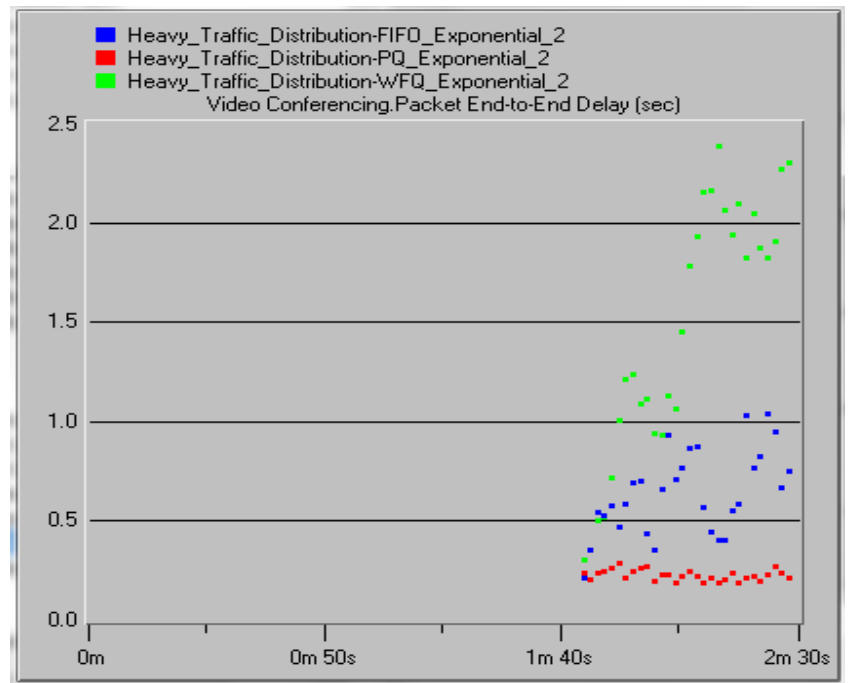

Figure 31. Packet end to end delay for 2 router configuration model.

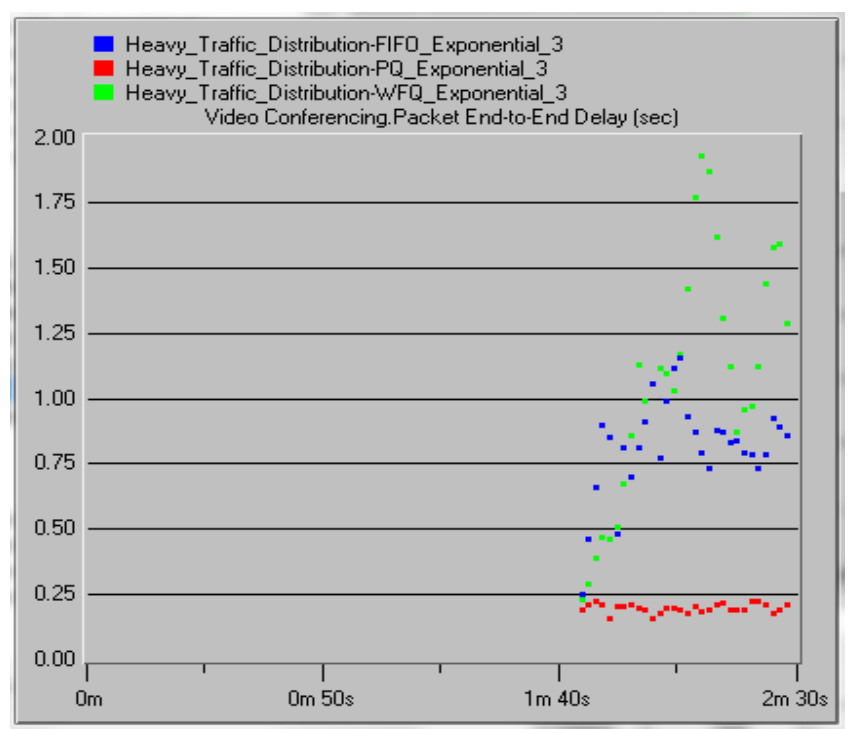

Figure 32. Packet end to end delay for 3 router configuration model. 


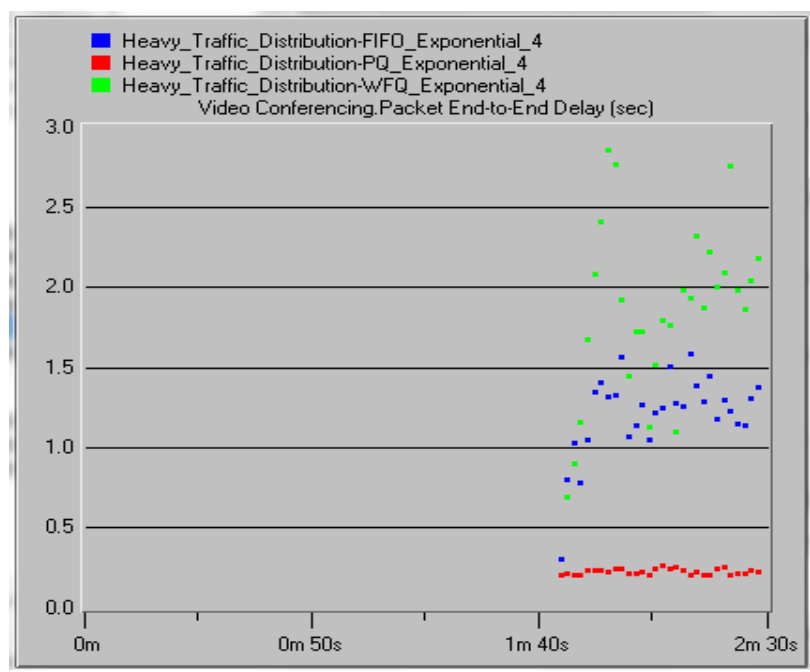

Figure 33. Packet end to end delay for 4 router configuration model.

Table 10. Statistics for Packet Delay Variation Exponential Distribution

\begin{tabular}{|c|c|c|c|}
\hline $\begin{array}{c}\text { Video } \\
\text { Applications }\end{array}$ & \multicolumn{2}{|c|}{$\begin{array}{c}\text { Packet Delay Variation (Sec) } \\
\text { PQ }\end{array}$} & FIFO \\
\hline 2 Routers & 0.12046 & 0.00722699 & 0.53762 \\
\hline 3 Routers & 0.283801 & 0.00758157 & 0.781538 \\
\hline 4 Routers & 1.22297 & 0.0156975 & 5.28668 \\
\hline
\end{tabular}

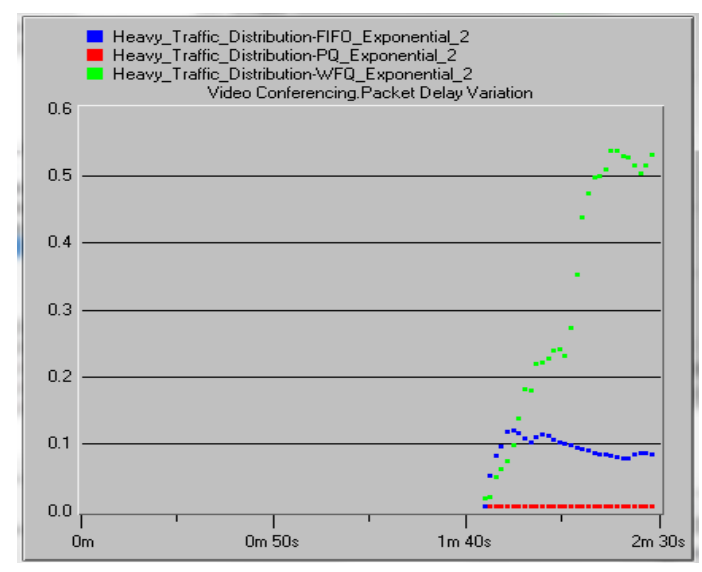

Figure 34. Packet delay variation for 2 router configuration model.

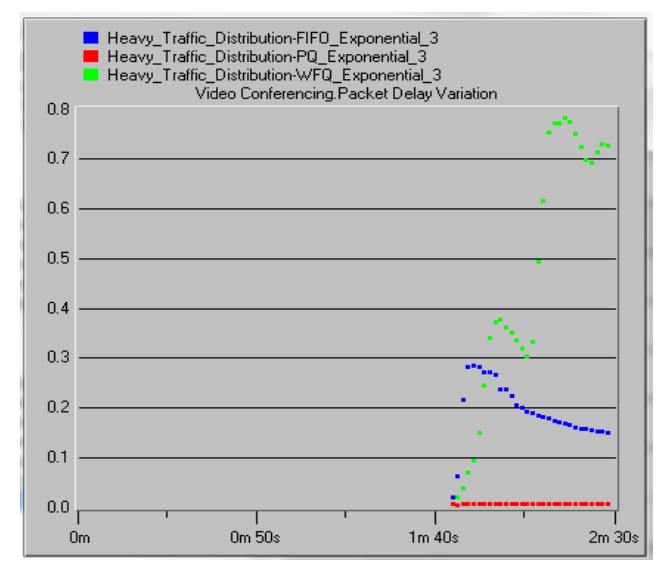

Figure 35. Packet delay variation for 3 router configuration model.

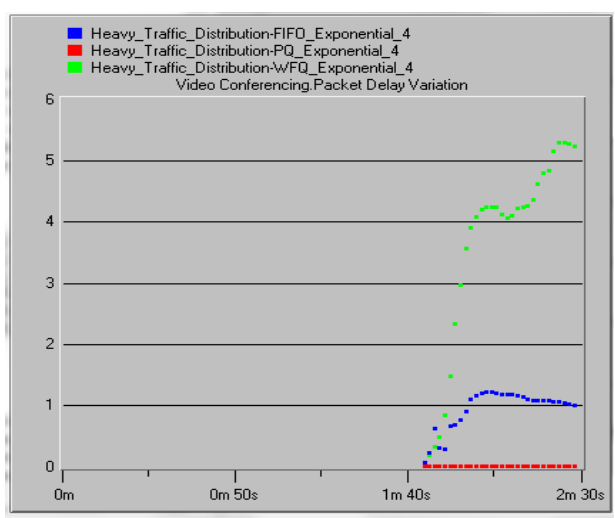

Figure 36. Packet delay variation for 4 router configuration model.

Packet delay variation is higher in case of WFQ scheme for video application. Fig 34, 35, 36 shows packet delay variation of Video transmission for two router, three router and four router configuration models. Packet delay variation is nearly zero for PQ scheme.

\section{CONCLUSION}

Generally internet traffic is bursty in nature. Due to this reason in this current research work two distributions uniform and exponential has been consider which generate bursty data. It has been observed after comparing the detail statistics of the result that packet end to end delay, traffic drop and packet delay variation is always higher in case of FIFO scheme for both voice and video based content delivery over network. For voice application PQ and WFQ schemes produces acceptable results whereas for video application PQ scheme proves to be better.

\section{ACKNOWLEDGEMENT}

Our sincere thanks to Thakur educational trust and management to provide all the facilities and infrastructure to carried out the research work.

\section{REFERENCES}

[1] Soung C. Liew, Tony T. Lee" A Fundamental Property for Traffic Management in ATM Networks" IEEE procedding 1993 Pages 12401249

[2] Pascal Lorenz, "QOS in Next Generation Network. 26th International Conference Interfaces ITI 2004, June 7-10.

[3] Neill Weilkinson "Next Generation Network Services Technologies and Strategies" john willey and sons ltd pp 167-170.

[4] I Akyildiz, "Exact product form solution for queuing networs with blocking," IEEE Trans Comput.. 36(1), 122-25 1987.

[5] J.P Buzen "Computational algorithm for closed queuing networks with exponential servers" Commun. ACM [1973] pp 527-531

[6] P.J Burke,"output of a queuing system" page(s): 699-704.

[7] "FIFO (First in First out)", http://www.daxnetworks.com/Technology/TechDost/TD-032206.pdf

[8] Taddia, C.; Mazzini, G.; On the Jitter Performance of FIFO and Priority Queues Mixture IEEE 17th International Symposium on 2006, Page(s): $1-5$

[9] Balogh, T.; Medvecky, M.; "Performance evaluation of WFQ, WF2Q+ and WRR queue scheduling algorithms ",Telecommunications and Signal Processing (TSP), 2011 34th International Conference 2011 , Page(s): 136 - 140

[10] Network World,"Weighted Fair Queuing" http://www.networkworld.com/details/659.html 
[11] Mong-Fong Homg; Wei-Tsong Lee; Kuan-Rong Lee; Yau-Hwang Kuo; An adaptive approach to weighted fair queue with QoS enhanced on IP network Proceedings of IEEE Region 10 International Conference on Volume: 12001 , Page(s): $181-186$

[12] G Bolch,S.Greiner,H.De Meer "Modelling and Performance evaluation with computer science Applications. Wiley, New York 1998.

[13] Kumar, S.; Kumar, P.R.; Performance bounds for queueing networks and scheduling policies IEEE Transactions Volume 39, Issue 8, Aug. 1994 pp 1600 - 1611

[14] B.R haverkort, R Marie, G Rubino and K.S Trivedi, “ Performability Modelling: Techniques and Tools, Wiley, New York,2001

[15] I Akyildiz, "Exact product form solution for queuing networs with blocking," IEEE Trans Comput.. 36(1), 122-25 1987.

[16] Mohammad Mirza Golam Rashed and Mamun Kabir" a comparative study of different queuing techniques in voip, video conferencing and file transfer" daffodil international university journal of science and technology, volume 5, issue 1, January 2010 pp 37-47.

\section{AUTHORS PROFILE}

Dr.B.K.Mishra was born in the year 1966 and has completed his Bachelor of
Engineering in Electronics from Amravati University in the year 1988, M.E. in Electronics and Communication Engineering in the year 1992 and Ph. D Engineering in Electronics \& Communication Engineering in 1998 from Birla Institute of Technology, Ranchi. He is having a teaching experience of more than 18 years with research experience of 4 years. He has published more than 20 technical papers in the National and International Journals including the proceedings of National and International conferences. He has also published six text books in the area of Electronics Devices and Circuits and Communication. The area of interest includes the Communication Engineering and Electronic Circuits. Currently he is working as a Principal of Thakur College of Engineering \& Technology since $12^{\text {th }}$ June' 06 .

S.K Singh is Head of Information technology department of Thakur College of Science and Commerce affiliated to University of Mumbai. He has received his M.E degree in Information Technology in 2006. He is having a teaching experience of more than 10 years at UG and PG level and currently he is research scholar in NMIMS University-Mumbai and his research interest include quality of service, next generation network and Packet inspection. He has published more than 10 technical papers in the National and International Journals including the proceedings of National and International conferences.

Kalpana Patel is Post Graduate Student in Thakur College of Science and Commerce affiliated to University of Mumbai. Her research interest include Networks, Mobile Communication, Next Generation Network and Distributed Computing. 